\title{
Refinements of Hermite-Hadamard inequality for operator convex function
}

Junmin $\mathrm{Han}^{\mathrm{a}, *}$, Jian Shi ${ }^{\mathrm{b}}$

a School of Mathematics and Information Science, Weifang University, Weifang, 261061, P. R. China.

${ }^{b}$ College of Mathematics and Information Science, Hebei University, Baoding, 071002, P. R. China.

Communicated by J. Brzdek

\begin{abstract}
In this paper, we present several operator versions of the Hermite-Hadamard inequality for the operator convex function, which are refinements of some operator convex inequalities presented by Dragomir [S. S. Dragomir, Appl. Math. Comput., 218 (2011), 766-772] and [S. S. Dragomir, RGMIA Research Report Collection, 2016 (2016), 15 pages]. (C)2017 All rights reserved.
\end{abstract}

Keywords: Self-adjoint operators, Hermite-Hadamard inequality, operator convex functions. 2010 MSC: 47A63.

\section{Introduction}

The following classical inequality, which was first discovered by Hermite in 1883 in the journal Mathesis (see [10]) and independently proved in 1893 by Hadamard in [9], is well-known as the HermiteHadamard inequality in the literature:

$$
f\left(\frac{a+b}{2}\right) \leqslant \frac{1}{b-a} \int_{a}^{b} f(t) d t \leqslant \frac{f(a)+f(b)}{2},
$$

where $f$ is a convex function on an interval $[a, b]$. Both inequalities hold in the reversed direction if $f$ is concave.

For any convex function defined on a segment $[a, b]$, one can easily observe that (1.1) is equivalent to the following double inequality:

$$
f\left(\frac{a+b}{2}\right) \leqslant \int_{0}^{1} f[(1-t) a+t b] d t \leqslant \frac{f(a)+f(b)}{2} .
$$

We note that Hermite-Hadamard inequality, regarded as a refinement of the concept of convexity, has several applications in nonlinear analysis and the geometry of Banach spaces. Hermite-Hadamard inequality for convex functions has received renewed attention in recent years and a remarkable variety of refinements and generalizations have been found (see, for example, [2, 6, 12]).

A real-valued continuous function $f$ on an interval $I$ is said to be operator convex if

$$
f((1-\lambda) A+\lambda B) \leqslant(1-\lambda) f(A)+\lambda f(B),
$$

in the operator order, for all $\lambda \in[0,1]$ and for self-adjoint operators $A$ and $B$ on a Hilbert space $H$ whose

\footnotetext{
${ }^{*}$ Corresponding author

Email addresses: goodlucktotoro@126.com (Junmin Han), mathematic@126.com (Jian Shi)
} 
spectra are contained in I.

In 2011, Dragomir [4] established the following Hermite-Hadamard type inequality for operator convex function.

Theorem 1.1. Let $\mathrm{f}: \mathrm{I} \rightarrow \mathbb{R}$ be an operator convex function on the interval $\mathrm{I}$. Then for any self-adjoint operators $A$ and $B$ with spectra in I we have the inequality

$$
\begin{aligned}
f\left(\frac{A+B}{2}\right) & \leqslant \frac{1}{2}\left[f\left(\frac{3 A+B}{4}\right)+f\left(\frac{A+3 B}{4}\right)\right] \\
& \leqslant \int_{0}^{1} f((1-t) A+t B) d t \leqslant \frac{1}{2}\left[f\left(\frac{A+B}{2}\right)+\frac{f(A)+f(B)}{2}\right] \leqslant \frac{f(A)+f(B)}{2} .
\end{aligned}
$$

In 2016, Dragomir [5] gave a refinement of the above Hermite-Hadamard inequality for operator convex function (1.2) as follows.

Theorem 1.2. Let $\mathrm{f}: \mathrm{I} \rightarrow \mathbb{R}$ be an operator convex function on the interval $\mathrm{I}$. Then for any self-adjoint operators $\mathrm{A}$ and $\mathrm{B}$ with spectra in $\mathrm{I}$ and for any $\lambda \in[0,1]$ we have the inequality

$$
\begin{aligned}
f\left(\frac{A+B}{2}\right) & \leqslant(1-\lambda) f\left[\frac{(1-\lambda) A+(1+\lambda) B}{2}\right]+\lambda f\left[\frac{(2-\lambda) A+\lambda B}{2}\right] \\
& \leqslant \int_{0}^{1} f((1-t) A+t B) d t \leqslant \frac{1}{2}[f((1-\lambda) A+\lambda B)+(1-\lambda) f(B)+\lambda f(A)] \leqslant \frac{f(A)+f(B)}{2} .
\end{aligned}
$$

For recent related results on Hermite-Hadamard type operator inequality, see $[1,8,11]$.

In this paper, we present several operator versions of the Hermite-Hadamard inequality for the operator convex function, which are refinements of operator convex inequalities (1.2) and (1.3).

\section{Main results}

For the first and the second inequalities in the Hermite-Hadamard inequality (1.1), Feng and Burqan constructed the following refinements, respectively.

Lemma 2.1 ([7]). Let $\mathrm{f}$ be a real-valued function which is convex on the interval $[\mathrm{a}, \mathrm{b}]$. Then

$$
f\left(\frac{a+b}{2}\right) \leqslant \frac{1}{b-a} \int_{a}^{b} f(t) d t \leqslant \frac{1}{4}\left[f(a)+2 f\left(\frac{a+b}{2}\right)+f(b)\right] \leqslant \frac{f(a)+f(b)}{2} .
$$

Lemma 2.2 ([3]). Let $\mathrm{f}$ be a real-valued function which is convex on the interval $[\mathrm{a}, \mathrm{b}]$. Then

$$
f\left(\frac{a+b}{2}\right) \leqslant \frac{1}{2}\left[f\left(\frac{3 a+b}{4}\right)+f\left(\frac{a+3 b}{4}\right)\right] \leqslant \frac{1}{b-a} \int_{a}^{b} f(t) d t \leqslant \frac{f(a)+f(b)}{2} .
$$

We present a refinement of the above Hermite-Hadamard inequality (2.1) and (2.2) as follows.

Lemma 2.3. Let $\mathrm{f}$ be a real-valued function which is convex on the interval $[\mathrm{a}, \mathrm{b}]$ and let $\mathrm{n}$ be a positive integer. Then

$$
\begin{aligned}
f\left(\frac{a+b}{2}\right) & \leqslant \frac{1}{2}\left\{f\left[\frac{\left(2^{n}+1\right) a+\left(2^{n}-1\right) b}{2^{n+1}}\right]+f\left[\frac{\left(2^{n}-1\right) a+\left(2^{n}+1\right) b}{2^{n+1}}\right]\right\} \\
& \leqslant \frac{1}{b-a} \int_{a}^{b} f(t) d t \leqslant \frac{1}{2(n+1)}\left[n f(a)+2 f\left(\frac{a+b}{2}\right)+n f(b)\right] \leqslant \frac{f(a)+f(b)}{2} .
\end{aligned}
$$

Proof. Since $f$ is convex on $[a, b]$, we have

$$
f\left(\frac{a+b}{2}\right) \leqslant \frac{f(a)+f(b)}{2} .
$$


Thus

$$
\frac{1}{2(n+1)}\left[n f(a)+2 f\left(\frac{a+b}{2}\right)+n f(b)\right] \leqslant \frac{1}{2(n+1)}[n f(a)+f(a)+f(b)+n f(b)]=\frac{f(a)+f(b)}{2} .
$$

This completes the proof of the fourth inequality.

Using Hermite-Hadamard inequality, we have

$$
\begin{aligned}
f\left(\frac{a+b}{2}\right) & =f\left[\frac{1}{2} \cdot \frac{\left(2^{n}+1\right) a+\left(2^{n}-1\right) b}{2^{n+1}}+\frac{1}{2} \cdot \frac{\left(2^{n}-1\right) a+\left(2^{n}+1\right) b}{2^{n+1}}\right] \\
& \leqslant \frac{1}{2}\left\{f\left[\frac{\left(2^{n}+1\right) a+\left(2^{n}-1\right) b}{2^{n+1}}\right]+f\left[\frac{\left(2^{n}-1\right) a+\left(2^{n}+1\right) b}{2^{n+1}}\right]\right\} .
\end{aligned}
$$

This completes the proof of the first inequality.

To prove the second and the third inequalities, it is only needed to prove the following inequalities by Lemmas 2.1 and 2.2:

$$
f\left[\frac{\left(2^{n}+1\right) a+\left(2^{n}-1\right) b}{2^{n+1}}\right]+f\left[\frac{\left(2^{n}-1\right) a+\left(2^{n}+1\right) b}{2^{n+1}}\right] \leqslant f\left(\frac{3 a+b}{4}\right)+f\left(\frac{a+3 b}{4}\right)
$$

and

$$
\frac{1}{2}\left[f(a)+2 f\left(\frac{a+b}{2}\right)+f(b)\right] \leqslant \frac{1}{n+1}\left[n f(a)+2 f\left(\frac{a+b}{2}\right)+n f(b)\right] .
$$

Next, we prove inequality (2.3) by induction. By Lemma 2.2, we have

$$
f(a)+f(b) \geqslant f\left(\frac{3 a+b}{4}\right)+f\left(\frac{a+3 b}{4}\right) .
$$

So (2.3) holds trivially for the case $n=1$. Now suppose the assertion (2.3) holds for the case $n=k$. By the induction hypothesis, we have

$$
\begin{aligned}
& f\left(\frac{3 a+b}{4}\right)+f\left(\frac{a+3 b}{4}\right) \\
& \quad \geqslant f\left[\frac{\left(2^{k}+1\right) a+\left(2^{k}-1\right) b}{2^{k+1}}\right]+f\left[\frac{\left(2^{k}-1\right) a+\left(2^{k}+1\right) b}{2^{k+1}}\right] \\
& \quad \geqslant f\left[\frac{\left.3 \cdot \frac{\left(2^{k}+1\right) a+\left(2^{k}-1\right) b}{2^{k+1}}+\frac{\left(2^{k}-1\right) a+\left(2^{k}+1\right) b}{2^{k+1}}\right]+f\left[\frac{\frac{\left(2^{k}+1\right) a+\left(2^{k}-1\right) b}{2^{k+1}}+3 \cdot \frac{\left(2^{k}-1\right) a+\left(2^{k}+1\right) b}{2^{k+1}}}{4}\right]}{4}\right]+f\left[\frac{\left(2^{k+1}-1\right) a+\left(2^{k+1}+1\right) b}{2^{(k+1)+1}}\right] .
\end{aligned}
$$

Hence, (2.3) holds for the case $n=k+1$. Similarly, the inequality (2.4) holds by induction. Given all that, the proof of Lemma 2.3 is complete.

Remark 2.4. When $n=1$, it is easy to see that Lemma 2.1 and Lemma 2.2 are special cases of Lemma 2.3.

By Lemma 2.3, we obtain our first refinement of Hermite-Hadamard inequality for operator convex function.

Theorem 2.5. Let $\mathrm{f}: \mathrm{I} \rightarrow \mathbb{R}$ be an operator convex function on the interval $\mathrm{I}$ and let $\mathrm{n}$ be a positive integer. Then for any self-adjoint operators $\mathrm{A}$ and $\mathrm{B}$ on a Hilbert space $\mathrm{H}$ with spectra in $\mathrm{I}$ we have the inequality

$$
\begin{aligned}
f\left(\frac{A+B}{2}\right) & \leqslant \frac{1}{2}\left\{f\left[\frac{\left(2^{n}+1\right) A+\left(2^{n}-1\right) B}{2^{n+1}}\right]+f\left[\frac{\left(2^{n}-1\right) A+\left(2^{n}+1\right) B}{2^{n+1}}\right]\right\} \\
& \leqslant \int_{0}^{1} f((1-t) A+t B) d t \leqslant \frac{1}{2(n+1)}\left[n f(A)+2 f\left(\frac{A+B}{2}\right)+n f(B)\right] \leqslant \frac{f(A)+f(B)}{2} .
\end{aligned}
$$


Proof. Let $x \in \mathrm{H}$ be a unit vector and two self-adjoint operators $A$ and $B$ with spectra in I. Define the real-valued function $\rho(t)=\langle f((1-t) A+t B) x, x\rangle$ on the interval $[0,1]$. Since $f$ is operator convex, then for any $t_{1}, t_{2} \in[0,1]$ and $\alpha, \beta \geqslant 0$ with $\alpha+\beta=1$ we have

$$
\begin{aligned}
\rho\left(\alpha t_{1}+\beta t_{2}\right) & =\left\langle f\left(\left(1-\left(\alpha t_{1}+\beta t_{2}\right)\right) A+\left(\alpha t_{1}+\beta t_{2}\right) B\right) x, x\right\rangle \\
& =\left\langle f\left(\alpha\left[\left(1-t_{1}\right) A+t_{1} B\right]+\beta\left[\left(1-t_{2}\right) A+t_{2} B\right]\right) x, x\right\rangle \\
& \leqslant \alpha\left\langle f\left(\left(1-t_{1}\right) A+t_{1} B\right) x, x\right\rangle+\beta\left\langle f\left(\left(1-t_{2}\right) A+t_{2} B\right) x, x\right\rangle=\alpha \rho\left(t_{1}\right)+\beta \rho\left(t_{2}\right) .
\end{aligned}
$$

So $\rho(t)$ is a convex function on $[0,1]$. Applying Lemma 2.3 to the convex function $\rho(t)$ on $[0,1]$, we have

$$
\begin{aligned}
\rho\left(\frac{1}{2}\right) & \leqslant \frac{1}{2}\left[\rho\left(\frac{2^{n}-1}{2^{n+1}}\right)+\rho\left(\frac{2^{n}+1}{2^{n+1}}\right)\right] \\
& \leqslant \int_{0}^{1} \rho(t) d t \leqslant \frac{1}{2(n+1)}\left[n \rho(0)+2 \rho\left(\frac{1}{2}\right)+n \rho(1)\right] \leqslant \frac{\rho(0)+\rho(1)}{2} .
\end{aligned}
$$

Hence

$$
\begin{aligned}
\left\langle f\left(\frac{A+B}{2}\right) x, x\right\rangle & \leqslant \frac{1}{2}\left\langle\left\{f\left[\frac{\left(2^{n}+1\right) A+\left(2^{n}-1\right) B}{2^{n+1}}\right]+f\left[\frac{\left(2^{n}-1\right) A+\left(2^{n}+1\right) B}{2^{n+1}}\right]\right\} x, x\right\rangle \\
& \leqslant \int_{0}^{1}\langle f((1-t) A+t B) x, x\rangle d t \\
& \leqslant \frac{1}{2(n+1)}\left\langle\left[n f(A)+2 f\left(\frac{A+B}{2}\right)+n f(B)\right] x, x\right\rangle \leqslant \frac{1}{2}\langle[f(A)+f(B)] x, x\rangle .
\end{aligned}
$$

Finally, since by the continuity of the function $f$ we have

$$
\int_{0}^{1}\langle f((1-t) A+t B) x, x\rangle d t=\left\langle\int_{0}^{1} f((1-t) A+t B) d t x, x\right\rangle
$$

for any $x \in H,\|x\|=1$ and any self-adjoint operators $A$ and B with spectra in I. Now (2.7) and (2.8) yield the whole inequalities (2.6) as desired.

Remark 2.6. Theorem 1.1 is a special case of Theorem 2.5 when $n=1$.

Corollary 2.7. Under the assumptions of Theorem 2.5 , if $\mathrm{n}=2$, then

$$
\begin{aligned}
f\left(\frac{A+B}{2}\right) & \leqslant \frac{1}{2}\left[f\left(\frac{5 A+3 B}{8}\right)+f\left(\frac{3 A+5 B}{8}\right)\right] \\
& \leqslant \int_{0}^{1} f((1-t) A+t B) d t \leqslant \frac{1}{3}\left[f(A)+f\left(\frac{A+B}{2}\right)+f(B)\right] \leqslant \frac{f(A)+f(B)}{2} .
\end{aligned}
$$

The following representation result is useful in the sequel.

Lemma 2.8 ([5]). Let $\mathrm{f}: \mathrm{I} \rightarrow \mathbb{C}$ be a continuous function on the interval $\mathrm{I}$ and two self-adjoint operators $\mathrm{A}$ and $\mathrm{B}$ on a Hilbert space $\mathrm{H}$ with spectra in $\mathrm{I}$. Then for any $\lambda \in[0,1]$ we have the representation

$$
\begin{aligned}
\int_{0}^{1} f((1-t) A+t B) d t= & (1-\lambda) \int_{0}^{1} f[(1-t)((1-\lambda) A+\lambda B)+t B] d t \\
& +\lambda \int_{0}^{1} f[(1-t) A+t((1-\lambda) A+\lambda B)] d t
\end{aligned}
$$


Theorem 2.9. Let $\mathrm{f}: \mathrm{I} \rightarrow \mathbb{R}$ be an operator convex function on the interval $\mathrm{I}$ and let $\mathrm{n}$ be a positive integer. Then for any self-adjoint operators $\mathrm{A}$ and $\mathrm{B}$ on a Hilbert space $\mathrm{H}$ with spectra in I we have the inequality

$$
\begin{aligned}
f\left(\frac{A+B}{2}\right) \leqslant & (1-\lambda) f\left[\frac{(1-\lambda) A+(1+\lambda) B}{2}\right]+\lambda f\left[\frac{(2-\lambda) A+\lambda B}{2}\right] \\
\leqslant & \frac{1-\lambda}{2}\left\{f\left[\frac{\left(2^{n}+1\right)(1-\lambda) A+\left[\left(2^{n}+1\right) \lambda+\left(2^{n}-1\right)\right] B}{2^{n+1}}\right]\right. \\
& \left.+f\left[\frac{\left(2^{n}-1\right)(1-\lambda) A+\left[\left(2^{n}-1\right) \lambda+\left(2^{n}+1\right)\right] B}{2^{n+1}}\right]\right\} \\
& +\frac{\lambda}{2}\left\{f\left[\frac{\left[\left(2^{n}-1\right)(1-\lambda)+\left(2^{n}+1\right)\right] A+\left(2^{n}-1\right) \lambda B}{2^{n+1}}\right]\right. \\
& \left.+f\left[\frac{\left[\left(2^{n}+1\right)(1-\lambda)+\left(2^{n}-1\right)\right] A+\left(2^{n}+1\right) \lambda B}{2^{n+1}}\right]\right\} \\
\leqslant & \int_{0}^{1} f((1-t) A+t B) d t \\
\leqslant & \frac{1}{2(n+1)}\{\lambda n f(A)+(1-\lambda) n f(B)+n f[(1-\lambda) A+\lambda B] \\
& \left.+2(1-\lambda) f\left[\frac{(1-\lambda) A+(1+\lambda) B}{2}\right]+2 \lambda f\left[\frac{(2-\lambda) A+\lambda B}{2}\right]\right\} \\
\leqslant & \frac{1}{2}\{f[(1-\lambda) A+\lambda B]+(1-\lambda) f(B)+\lambda f(A)\} \leqslant \frac{f(A)+f(B)}{2} .
\end{aligned}
$$

Proof. Using the Hermite-Hadamard inequality (2.6) we have

$$
\begin{aligned}
f\left[\frac{(1-\lambda) A+(1+\lambda) B}{2}\right] \leqslant & \frac{1}{2}\left\{f\left[\frac{\left(2^{n}+1\right)(1-\lambda) A+\left[\left(2^{n}+1\right) \lambda+\left(2^{n}-1\right)\right] B}{2^{n+1}}\right]\right. \\
& \left.+f\left[\frac{\left(2^{n}-1\right)(1-\lambda) A+\left[\left(2^{n}-1\right) \lambda+\left(2^{n}+1\right)\right] B}{2^{n+1}}\right]\right\} \\
& \leqslant \int_{0}^{1} f[(1-t)((1-\lambda) A+\lambda B)+t B] d t \\
& \leqslant \frac{1}{2(n+1)}\left\{n f[(1-\lambda) A+\lambda B]+2 f\left[\frac{(1-\lambda) A+(1+\lambda) B}{2}\right]+n f(B)\right\} \\
& \leqslant \frac{f[(1-\lambda) A+\lambda B]+f(B)}{2} .
\end{aligned}
$$

and

$$
\begin{aligned}
f\left[\frac{(2-\lambda) A+\lambda B}{2}\right] \leqslant & \frac{1}{2}\left\{f\left[\frac{\left[\left(2^{n}-1\right)(1-\lambda)+\left(2^{n}+1\right)\right] A+\left(2^{n}-1\right) \lambda B}{2^{n+1}}\right]\right. \\
& \left.+f\left[\frac{\left[\left(2^{n}+1\right)(1-\lambda)+\left(2^{n}-1\right)\right] A+\left(2^{n}+1\right) \lambda B}{2^{n+1}}\right]\right\} \\
\leqslant & \int_{0}^{1} f[(1-t) A+t((1-\lambda) A+\lambda B)] d t \\
\leqslant & \frac{1}{2(n+1)}\left\{n f(A)+2 f\left[\frac{(2-\lambda) A+\lambda B}{2}\right]+n f[(1-\lambda) A+\lambda B]\right\} \\
& \leqslant \frac{f(A)+f[(1-\lambda) A+\lambda B]}{2} .
\end{aligned}
$$

If we multiply inequality (2.11) by $1-\lambda$ and (2.12) by $\lambda$, add the obtained inequalities, and use representa- 
tion (2.9), then we have

$$
\begin{aligned}
(1-\lambda) f & {\left[\frac{(1-\lambda) A+(1+\lambda) B}{2}\right]+\lambda f\left[\frac{(2-\lambda) A+\lambda B}{2}\right] } \\
\leqslant & \frac{1-\lambda}{2}\left\{f\left[\frac{\left(2^{n}+1\right)(1-\lambda) A+\left[\left(2^{n}+1\right) \lambda+\left(2^{n}-1\right)\right] B}{2^{n+1}}\right]\right. \\
& \left.+f\left[\frac{\left(2^{n}-1\right)(1-\lambda) A+\left[\left(2^{n}-1\right) \lambda+\left(2^{n}+1\right)\right] B}{2^{n+1}}\right]\right\} \\
& +\frac{\lambda}{2}\left\{f\left[\frac{\left[\left(2^{n}-1\right)(1-\lambda)+\left(2^{n}+1\right)\right] A+\left(2^{n}-1\right) \lambda B}{2^{n+1}}\right]\right. \\
& \left.+f\left[\frac{\left[\left(2^{n}+1\right)(1-\lambda)+\left(2^{n}-1\right)\right] A+\left(2^{n}+1\right) \lambda B}{2^{n+1}}\right]\right\} \\
\leqslant & \int_{0}^{1} f((1-t) A+t B) d t \\
\leqslant & \frac{1}{2(n+1)}\{\lambda n f(A)+(1-\lambda) n f(B)+n f[(1-\lambda) A+\lambda B] \\
& \left.+2(1-\lambda) f\left[\frac{(1-\lambda) A+(1+\lambda) B}{2}\right]+2 \lambda f\left[\frac{(2-\lambda) A+\lambda B}{2}\right]\right\} \\
\leqslant & (1-\lambda) \frac{f[(1-\lambda) A+\lambda B]+f(B)}{2}+\lambda \frac{f(A)+f[(1-\lambda) A+\lambda B]}{2},
\end{aligned}
$$

which proves the inequality in (2.10) except the first and last inequalities. By the operator convexity of $f$ we have

$$
\begin{aligned}
& (1-\lambda) f\left[\frac{(1-\lambda) A+(1+\lambda) B}{2}\right]+\lambda f\left[\frac{(2-\lambda) A+\lambda B}{2}\right] \\
& \quad \geqslant f\left[(1-\lambda) \frac{(1-\lambda) A+(1+\lambda) B}{2}+\lambda \frac{(2-\lambda) A+\lambda B}{2}\right]=f\left(\frac{A+B}{2}\right)
\end{aligned}
$$

and

$$
\frac{1}{2}\{f[(1-\lambda) A+\lambda B]+(1-\lambda) f(B)+\lambda f(A)\} \leqslant \frac{1}{2}\{(1-\lambda) f(A)+\lambda f(B)+(1-\lambda) f(B)+\lambda f(A)\}=\frac{f(A)+f(B)}{2}
$$

which proves the first and last inequalities in (2.10). So Theorem 2.9 is proved.

Corollary 2.10. Under the assumptions of Theorem 2.9, if $\mathrm{n}=1$, then

$$
\begin{aligned}
f\left(\frac{A+B}{2}\right) \leqslant & (1-\lambda) f\left[\frac{(1-\lambda) A+(1+\lambda) B}{2}\right]+\lambda f\left[\frac{(2-\lambda) A+\lambda B}{2}\right] \\
\leqslant & \frac{1-\lambda}{2}\left\{f\left[\frac{3(1-\lambda) A+(1+3 \lambda) B}{4}\right]+f\left[\frac{(1-\lambda) A+(3+\lambda) B}{4}\right]\right\} \\
& +\frac{\lambda}{2}\left\{f\left[\frac{(4-\lambda) A+\lambda B}{4}\right]+f\left[\frac{(4-3 \lambda) A+3 \lambda B}{4}\right]\right\} \\
\leqslant & \int_{0}^{1} f((1-t) A+t B) d t
\end{aligned}
$$




$$
\begin{aligned}
\leqslant & \frac{1}{4}\left\{\lambda f(A)+(1-\lambda) f(B)+f[(1-\lambda) A+\lambda B]+2 \lambda f\left[\frac{(2-\lambda) A+\lambda B}{2}\right]\right. \\
& \left.+2(1-\lambda) f\left[\frac{(1-\lambda) A+(1+\lambda) B}{2}\right]\right\} \\
\leqslant & \frac{1}{2}\{f[(1-\lambda) A+\lambda B]+(1-\lambda) f(B)+\lambda f(A)\} \leqslant \frac{f(A)+f(B)}{2} .
\end{aligned}
$$

Remark 2.11. Theorem 2.9 and Corollary 2.10 are refinements of Theorem 1.2.

\section{Acknowledgment}

The authors sincerely thank referee for his/her valuable comments. The project is supported by Natural Science Foundation of Shandong Province (No. BS2015SF006) and National Natural Science Foundation of China, Tian Yuan Foundation (No. 11226087).

\section{References}

[1] V. Bacak, R. Türkmen, Refinements of Hermite-Hadamard type inequalities for operator convex functions, J. Inequal. Appl., 2013 (2013), 10 pages. 1

[2] N. S. Barnett, P. Cerone, S. S. Dragomir, Some new inequalities for Hermite-Hadamard divergence in information theory, Stochastic analysis and applications, Stoch. Anal. Appl., 3 2002, 7-19. 1

[3] A. Burqan, Improved Cauchy-Schwarz norm inequality for operators, J. Math. Inequal., 10 (2016), 205-211. 2.2

[4] S. S. Dragomir, Hermite-Hadamard type inequalities for operator convex functions, Appl. Math. Comput., 218 (2011), 766-772. 1

[5] S. S. Dragomir, Some Hermite-Hadamard Type Inequalities for Operator Convex Functions and Positive Maps, RGMIA Research Report Collection, 2016 (2016), 15 pages. 1, 2.8

[6] S. S. Dragomir, C. E. M. Pearce, Selected Topics on Hermite-Hadamard Inequalities, RGMIA Monographs, Victoria University, (2000). 1

[7] Y. Feng, Refinements of the Heinz inequalities, J. Inequal. Appl., 2012 (2012), 6 pages. 2.1

[8] A. G. Ghazanfari, A. Barani, Some Hermite-Hadamard type inequalities for the product of two operator preinvex functions, Banach J. Math. Anal., 9 (2015), 9-20. 1

[9] J. Hadamard, Étude sur les propriétés des fonctions entiéres et en particulier d'une fonction considérée par Riemann (French), ?J. Math. Pures Appl., 58 (1893), 171-216. 1

[10] D. S. Mitrinović, I. B. Lacković, Hermite and convexity, Aequationes Math., 28 (1985), 229-232. 1

[11] M. S. Moslehian, Matrix Hermite-Hadamard type inequalities, Houston J. Math., 39 (2013), 177-189. 1

[12] C. P. Niculescu, L.-E. Persson, Convex functions and their applications: A contemporary approach, Springer, New York, (2006). 1 